\title{
Das Optimult-Studienkonzept - Grundlage für selektiven Einsatz neoadjuvanter Radiochemotherapie auf MRT-Basis?
}

\section{The Optimult Study Concept - Selective Neoadjuvant Chemoradiation Therapy Based on Preoperative MRI?}

Autoren

Institute
M. E. Kreis ${ }^{1}$, T. Junginger ${ }^{2}$, C. Rödel ${ }^{3}$, V. Heinemann ${ }^{4}$, K. Nikolaou ${ }^{5}$, U. Mansmann ${ }^{6}$, K. W. Jauch ${ }^{1}$

Die Institutsangaben sind am Ende des Beitrags gelistet.

\section{Erratum}

M. E. Kreis, T. Junginger, C. Rödel, V. Heinemann, K. Nikolaou, U. Mansmann, K. W. Jauch Das Optimult-Studienkonzept - Grundlage für selektiven Einsatz neoadjuvanter Radiochemotherapie auf MRT-Basis?

Zentralbl Chir 2010; 135: 302-306

Der Artikel enthält Fehler in der Schreibweise zweier Autoren.

Der Name des einen Autors muss V. Heinemann lauten, nicht V. Heinemanne.

Der Name des anderen Autors muss K. Nikolaou lauten, nicht Konstantin N. 